\title{
Improved Georeferencing: Three essential guiding documents
}

\author{
Arthur D Chapman $¥$, John R Wieczorek§, Paula F Zermoglio§, Maria Celeste Lunal, David A Bloom $\llbracket$ \\ $\ddagger$ Australian Biodiversity Information Services, Ballan, Australia \\ $\S$ VertNet, Bariloche, Argentina \\ | Museo Argentino de Ciencias Naturales "Bernardino Rivadavia"-CONICET, Buenos Aires, Argentina \\ II VertNet, Sebastopol, United States of America
}

\author{
Corresponding author: Arthur D Chapman (biodiv 2@achapman.org), \\ John R Wieczorek (gtuco.btuco@gmail.com), Paula F Zermoglio (pzermoglio@gmail.com) \\ Received: 25 Sep 2020 | Published: 29 Sep 2020 \\ Citation: Chapman AD, Wieczorek JR, Zermoglio PF, Luna MC, Bloom DA (2020) Improved Georeferencing: \\ Three essential guiding documents. Biodiversity Information Science and Standards 4: e58983. \\ https://doi.org/10.3897/biss.4.58983
}

\begin{abstract}
To understand biological and geological events and the history of collected samples, it is essential to determine and communicate location information accurately. The accuracy of a georeference depends upon the circumstances of the event. Historical collections depend on having clear verbatim locality descriptions, the correct interpretation of data written on labels, and on the availability of gazetteers and maps of suitable scale and time. Observation and tracking data localities depend on GPS (Global Positiioning System) accuracy, and on presence or absence of nearby obstructions such as buildings, forest cover, cliffs, etc. Marine data depend on the accurate determination of the surface location and the techniques to position a dive event from that location and to determine its depth and extent. Many people are using smartphones or maps such as Google Earth and Google Maps to determine their georeferences - but are they suitable and accurate enough to determine locations and elevations?
\end{abstract}

New editions of the Georeferencing Best Practices (Chapman and Wieczorek 2020), the Georeferencing Quick Reference Guide (Zermoglio et al. 2020), and the Georeferencing Calculator Manual (Bloom et al. 2020), were published earlier this year and address all the issues listed above and many more. These documents were based on earlier versions but 
have been updated and improved considerably - adding information for marine biomes, caves, lithographic stratifications, transects, and the use of elevation, as well as including many more illustrations and examples. The expansion of an extensive georeferencing glossary adds to consistency in the use of terms The trio of documents now provides consistent guidance about how to georeference diverse locality types and detailed instructions on how to calculate uncertainty using many different coordinate reference systems and datums (horizontal and vertical) along with the importance of recording this information. Finally, they provide guidance on how to set up a georeferencing project and how to relate the results to the Darwin Core Standard (Darwin Core Task Group 2009).

For the last decade, Darwin Core (Wieczorek et al. 2012) has been one of the preferred standards for sharing biodiversity data, including associated location information. Darwin Core currently has 44 terms in its Location class, allowing sharing from administrative divisions, to elevations and depths, coordinates in different formats, and georeference metadata, among others. Although Darwin Core provides definitions for each of its terms, their correct use is sometimes poorly understood, resulting in information being captured incorrectly, or not captured, documented or shared at all. We will re-introduce these documents, discuss their content, importance, and differences from previously published versions. The newly revised documents provide guidance on capturing and documenting georeferences, clarifying the georeferencing process and showing how to capture information using Darwin Core appropriately. They will improve the location data associated with biological events and our understanding of these events.

\section{Keywords}

biodiversity, georeferencing, documentation, methods

\section{Presenting author}

Arthur D Chapman

\section{Presented at}

TDWG 2020

\section{Funding program}

GBIF Documentation

\section{Hosting institution}

Global Biodiversity Information Facility 


\section{References}

- $\quad$ Bloom DA, Wieczorek J, Zermoglio PF (2020) Georeferencing Calculator Manual. GBIF Secretariat, Copenhagen. [In English]. https://doi.org/10.35035/gdwq-3v93

- Chapman A, Wieczorek J (2020) Georeferencing Best Practices. GBIF Secretariat, Copenhagen. [In English]. https://doi.org/10.15468/doc-gg7h-s853

- Darwin Core Task Group (2009) Darwin Core. http://www.tdwg.org/standards/450. Accessed on: 2020-9-13.

- Wieczorek J, Bloom D, Guralnick R, Blum S, Döring M, Giovanni R, (2012) Darwin Core: An Evolving Community-Developed Biodiversity Data Standard. PLoS ONE 7 (1). [In English]. https://doi.org/10.1371/journal.pone.0029715

- Zermoglio PF, Chapman AD, Wieczorek J, Luna M, Bloom DA (2020) Georeferencing Quick Reference Guide. GBIF Secretariat, Copenhagen. [In English]. https://doi.org/ $\underline{10.35035 / \mathrm{e} 09 \mathrm{p}-\mathrm{h} 128}$ 